\title{
A general relativistic Thomas-Fermi treatment of neutron star cores
}

\section{Pugliese, Jorge A. Rueda, R. Ruffini and S.-S. Xue}

Dipartimento di Fisica and ICRA, Sapienza Università di Roma

P.le Aldo Moro 5, I-00185 Rome, Italy and ICRANet, P.zza della Repubblica 10, I-65122

Pescara, Italy

E-mail: daniela.pugliese@ICRA.it, Rueda@icra.it, Ruffini@icra.it,

Xuedicra.it

We formulate the set of self-consistent ground-state equilibrium equations for neutron star cores taking into account quantum statistics, electro-weak, and strong interactions, within the framework of general relativity. The strong interaction between nucleons is modeled through the sigmaomega-rho meson exchange in the context of the extended Walecka model, all duly expressed in general relativity. We found the generalization to the works of Klein (1949), of Kodama and Yamada (1972), and of Olson and Baylin (1975) by demonstrating that the thermodynamic equilibrium condition of the constancy of the Fermi energy of each particle-specie can be properly generalized to include the contribution of all fields. The consequences of these new conditions of equilibrium on the structure of neutron stars are discussed.

25th Texas Symposium on Relativistic Astrophysics - TEXAS 2010

December 06-10, 2010

Heidelberg, Germany 


\section{Introduction}

In addition to the constancy of the temperature, thermodynamic equilibrium demands, in absence of any external field, the constancy of the particle chemical potential throughout the configuration. In presence of an external field, such a condition becomes [1] $\mu_{0}+U=$ constant, where $U$ denotes the external potential and $\mu_{0}$ is the free-particle chemical potential. The extension of these equilibrium conditions to the case of general relativity were obtained by O. Klein [2], who investigated the thermodynamic equilibrium conditions of a self-gravitating one-component fluid of non-interacting neutral particles in spherical symmetry. The generalization of the Klein's equilibrium conditions to the case of a multi-component fluid of non-interacting neutral particles was given by T. Kodama and M. Yamada [3]. E. Olson and M. Bailyn [4] went one step further obtaining the equilibrium conditions for a self-gravitating multi-component fluid of charged particles taking into account the Coulomb interaction. Having in mind the case of neutron star interiors, in this article we make a brief description of the generalization of the above works to include the strong interaction for the hadronic species and the Coulomb interaction for the charged species within a self-consistent general relativistic treatment. In particular, we assume neutron star cores composed of interacting degenerate neutrons, protons and electrons in beta equilibrium. Thus, we shall develop a general relativistic Thomas-Fermi treatment of neutron star cores within the framework of quantum statistics and of the general relativistic field theory for the gravitational, the electromagnetic and the hadronic fields. We consider the electromagnetic interaction between electrons and protons and, for the hadronic interaction, we follow the so-called Walecka model or quantum hadrodynamical model $[5,6]$, in which the strong interaction is modeled by mesonexchange through the sigma, omega and rho meson-fields. We adopt units with $\hbar=c=1$. The Latin indexes vary from 1 to 3, Greek indexes from 0 to 4 .

\section{General formulation}

The total lagrangian density of the system is given by

$$
\mathscr{L}=\mathscr{L}_{g}+\mathscr{L}_{f}+\mathscr{L}_{\sigma}+\mathscr{L}_{\omega}+\mathscr{L}_{\rho}+\mathscr{L}_{\gamma}+\mathscr{L}_{\text {int }},
$$

where $\sigma$ is an isoscalar meson field, providing the attractive nuclear force, $\omega$ is the massive vector field, modeling the repulsive short range nuclear force and $\rho$ is the massive isovector field that takes account of the surface effects of nuclei modeling a repulsive nuclear force $[5,6,7,8]$. Therefore the lagrangian densities for the free fields are

$$
\begin{array}{ll}
\mathscr{L}_{f}=\sum_{i=e, N} \bar{\psi}_{i}\left(r \gamma^{\mu} \partial_{\mu}-m_{i}\right) \psi_{i}, & \mathscr{L}_{\sigma}=\frac{1}{2} \nabla_{\mu} \sigma \nabla^{\mu} \sigma-U(\sigma), \\
\mathscr{L}_{\omega}=-\frac{1}{4} \Omega_{\mu \nu} \Omega^{\mu v}+\frac{1}{2} m_{\omega}^{2} \omega_{\mu} \omega^{\mu}, & \mathscr{L}_{\gamma}=-\frac{1}{4} F_{\mu v} F^{\mu v}, \\
\mathscr{L}_{\rho}=-\frac{1}{4} \mathscr{R}_{\mu \nu} \mathscr{R}^{\mu v}+\frac{1}{2} m_{\rho}^{2} \rho_{\mu} \rho^{\mu}, & \mathscr{L}_{g}=-\frac{R}{16 \pi G},
\end{array}
$$

where $\psi_{N}$ is the nucleon isospin doublet, $\psi_{e}$ is the electronic singlet, $m_{i}$ states for the mass of each particle-specie and $\Omega_{\mu v} \equiv \partial_{\mu} \omega_{v}-\partial_{v} \omega_{\mu}, \mathscr{R}_{\mu \nu} \equiv \partial_{\mu} \rho_{v}-\partial_{v} \rho_{\mu}, F_{\mu \nu} \equiv \partial_{\mu} A_{v}-\partial_{v} A_{\mu}$ the field 
strengths for the $\omega^{\mu}, \rho$ and $A^{\mu}$ fields respectively. $U(\sigma)$ denotes the self interaction scalar field potential, which is a quartic-order polynom for a renormalizable theory $[9,10,11]$, and $R$ is the Ricci scalar. The interacting part of the lagrangian density is

$$
\mathscr{L}_{\text {int }}=-g_{\sigma} \sigma \bar{\psi}_{N} \psi_{N}-g_{\omega} \omega_{\mu} J_{\omega}^{\mu}-g_{\rho} \rho_{\mu} J_{\rho}^{\mu}+e A_{\mu} J_{\gamma, e}^{\mu}-e A_{\mu} J_{\gamma, N}^{\mu},
$$

where the currents are $J_{\omega}^{\mu} \equiv \bar{\psi}_{N} \gamma^{\mu} \psi_{N}, J_{\rho}^{\mu} \equiv \bar{\psi}_{N} \tau_{3} \gamma^{\mu} \psi_{N}, J_{\gamma, e}^{\mu} \equiv \bar{\psi}_{e} \gamma^{\mu} \psi_{e}$ and $J_{\gamma, N}^{\mu} \equiv \bar{\psi}_{N}\left(\frac{1+\tau_{3}}{2}\right) \gamma^{\mu} \psi_{N}$ with a bar denoting usual Hermitian conjugation. The coupling constants of the $\sigma, \omega$ and $\rho$-fields are $g_{\sigma}, g_{\omega}$ and $g_{\rho}$, and $e$ is the fundamental electric charge. The Dirac matrices $\gamma^{\mu}$ and the isospin Pauli matrices satisfy the Dirac algebra in curved spacetime[12]. The energy-density $\mathscr{E}$ and the pressure $\mathscr{P}$ of the fermion fluid are

$$
\mathscr{E}=\frac{2}{(2 \pi)^{3}} \sum_{i=n, p, e} \int_{0}^{K_{i}^{F}} \varepsilon_{i} d^{3} k, \quad \mathscr{P}=\frac{2}{3(2 \pi)^{3}} \sum_{i=n, p, e} \int_{0}^{K_{i}^{F}} \frac{k^{2}}{\varepsilon_{i}} d^{3} k,
$$

where $\varepsilon_{i}=\sqrt{k^{2}+\left(\tilde{m}_{i}\right)^{2}}$ denotes the single-particle energy spectrum, for electrons $\tilde{m}_{e}=m_{e}$, and the nucleon effective mass is $\tilde{m}_{N} \equiv m_{N}+g_{\sigma} \sigma$, and $K_{i}^{F}$ denotes the Fermi momentum of each particle specie. where $\mu_{i}=\sqrt{\left(K_{i}^{F}\right)^{2}+\left(\tilde{m}_{i}\right)^{2}}$ and $n_{i}=\left(K_{i}^{F}\right)^{3} /\left(3 \pi^{2}\right)$ are the free-chemical potential and number density of the $i$-specie.

\section{Generalized Fermi energies and beta equilibrium}

The nucleon doublet and the electronic spinor written in the phase-space are $\psi_{i}=\psi_{i}(k) e^{-l k_{\mu} x^{\mu}}$. From the Dirac equations we obtain the following equations $\left(\gamma_{\mu} \mathscr{K}^{\mu}-\tilde{m}_{i}\right) \psi_{i}(k)=0$ with $\mathscr{K}^{\mu} \equiv$ $k^{\mu}-V_{i}^{\mu}, V_{e}=-e V$. Where $V_{i}^{\mu} \equiv g_{\omega} \omega^{\mu}+g_{\rho} \tau \rho^{\mu}+e\left(\frac{1+\tau_{3}}{2}\right) A^{\mu}$ is the effective four potential of nucleons. Making a quadrature of the Dirac operators in the phase-space we obtain the Fermi energy for electrons $E_{e}^{F}$, neutrons $E_{n}^{F}$ and protons $E_{p}^{F}$

$$
\begin{aligned}
& E_{e}^{F}=\sqrt{g_{00}} \mu_{e}-e V=e^{v / 2} \mu_{e}-e V, \\
& E_{n}^{F}=\sqrt{g_{00}} \mu_{n}+g_{\omega} \omega-g_{\rho} \rho=e^{v / 2} \mu_{n}+g_{\omega} \omega-g_{\rho} \rho, \\
& E_{p}^{F}=\sqrt{g_{00}} \mu_{p}+g_{\omega} \omega+g_{\rho} \rho+e V=e^{v / 2} \mu_{p}+g_{\omega} \omega+g_{\rho} \rho+e V .
\end{aligned}
$$

Consequently, the beta equilibrium condition $E_{n}^{F}=E_{p}^{F}+E_{e}^{F}$, becomes

$$
\mu_{n}=\mu_{p}+\mu_{e}+2 g_{\rho} \rho e^{-v / 2} .
$$

Where we use the notation $\omega_{0}=\omega, \rho_{0}=\rho$ and $A_{0}=V$, a prime stands for radial derivative,

\section{Constancy of the generalized Fermi energies}

Using the equations of motion for the fields $\rho, \omega$ and $\sigma$, the energy-momentum conservation and the Eqs. (2.4), the energy-momentum conservation equation can be rewritten as

$$
\sum_{i=n, p, e} n_{i} d\left(e^{v / 2} \mu_{i}\right)+g_{\omega} n_{b} d \omega+g_{\rho} n_{3} d \rho+e n_{c h} d V=0,
$$


where $n_{c h} \equiv\left(n_{p}-n_{e}\right)$, and $n_{b}, n_{p}, n_{n}$ and $n_{e}$ are the baryon, proton, neutron and electron number density.

Using the expressions (3.1)-(3.3) and the beta equilibrium condition (3.4), the Eq. (4.1) becomes

$$
\sum_{i=p, e}\left(n_{i}+n_{n}\right) d E_{i}^{F}=0
$$

It was demonstrated in the non interacting case[4] that from the minimization energy procedure it follows the thermodynamic energy condition of constancy of the generalized particle Fermi energy of all particle species. It can be seen from Eq. (4.2) that it is enough to request the constancy of the generalized electron Fermi energy

$$
E_{e}^{F}=e^{v / 2} \mu_{e}-e V=\text { constant },
$$

to obtain the constancy of $E_{p}^{F}$ and consequently, from beta equilibrium, the constancy of $E_{n}^{F}[13,14$, $15,16,17]$. Then, in addition to the electron equilibrium condition (4.3) we obtain for the nucleon components

$$
E_{n, p}^{F}=e^{v / 2} \mu_{n, p}+\mathscr{V}_{n, p}=\mathrm{constant}, \quad \mathscr{V}_{n, p} \equiv g_{\omega} \omega+g_{\rho} \tau \rho+e\left(\frac{1+\tau_{3}}{2}\right) V .
$$

\section{Conclusions}

We have presented a self-consistent treatment of self-gravitating system of degenerate neutrons, protons and electrons in beta equilibrium within the framework of general relativity including quantum statistics, electro-weak, and strong interactions. We obtained the generalized particle Fermi energies from the Dirac equations for nucleons and electrons.

Then, we used the generalized Fermi energies to obtain the modified beta equilibrium condition for the particle species. Finally, we outlined how from the Einstein-Maxwell-Dirac equations, the electron equilibrium condition and the beta equilibrium condition, it follows the constancy of the generalized particle Fermi energy of each particle specie including the contribution of all fields.

\section{References}

[1] L. D. Landau and E. M. Lifshitz. Statistical physics. Pt.1, Pergamon (1980).

[2] O. Klein, Rev. Mod. Phys. 21531 (1949).

[3] T. Kodama and M. Yamada, Prog. Theor. Phys. 47, 444 (1972).

[4] E. Olson and M. Bailyn, Phys. Rev. D 12 (1975) 3030.

[5] H. P. Duerr, Phys. Rev. 103 (1956) 469.

[6] J. D. Walecka, Annals Phys. 83 (1974) 491.

[7] J. Boguta and A. R. Bodmer, Nucl. Phys. A 292 (1977) 413.

[8] P. Ring, Prog. Part. Nucl. Phys. 37 (1996) 193.

[9] T. D. Lee and G. C. Wick, Phys. Rev. D 9 (1974) 2291. 
[10] T. D. Lee, Rev. Mod. Phys. 47 (1975) 267.

[11] T. D. Lee and M. Margulies, Phys. Rev. D 11 (1975) 1591

[12] T. D. Lee and Y. Pang, Phys. Rev. D 35 (1987) 3678.

[13] Jorge A. Rueda, R. Ruffini and S.-S. Xue, arXiv:0911.4627 submitted to Phys. Rev. Lett. (2010).

[14] Jorge A. Rueda, M. Rotondo, R. Ruffini and S.-S. Xue, submitted to Phys. Rev. Lett. (2010).

[15] Jorge A. Rueda, M. Rotondo, D. Pugliese, R. Ruffini and S.-S. Xue. submitted to Int. J. Mod. Phys. D.

[16] D. Pugliese, Jorge A. Rueda, M. Rotondo, R. Ruffini and S.-S. Xue. submitted to Int. J. Mod. Phys. D.

[17] D. Pugliese, Jorge A. Rueda, R. Ruffini and S.-S. Xue to be submitted to Phys. Rev. D. 